\title{
ON CONVERGENCE OF POLYNOMIAL SOLUTIONS OF MINIMAL SURFACE
}

\section{A.A. KLYACHIN, I.V.TRUHLYAEVA}

\begin{abstract}
In this paper we consider the polynomial approximate solutions of the Dirichlet problem for minimal surface equation. It is shown that under certain conditions on the geometric structure of the domain the absolute values of the gradients of the solutions are bounded as the degree of these polynomials increases. The obtained properties imply the uniform convergence of approximate solutions to the exact solution of the minimal surface equation.
\end{abstract}

Keywords: minimal surface equation, uniform convergence, approximate solution.

Mathematics Subject Classification: 35J25, 35J93, 65N30

\section{INTRODUCTION}

In numerical solving of boundary value problems for equations and systems of partial differential equations, a very important issue is the convergence of approximate solutions. The study of this issue is especially important for nonlinear equations since in this case there is a series of difficulties related with the impossibility of employing traditional methods and approaches used for linear equations. At present, a quite topical problem is to determine the conditions ensuring the uniform convergence of approximate solutions obtained by various methods for nonlinear equations and system of equations of variational kind. In this case, it is natural to employ variational methods of solving boundary value problems. And this is an issue on the justification of these methods arises, which is reduced to studying general properties of approximate solutions (see, for instance, [1], [2]).

\section{Formulation of the PROBlem}

We consider the issue on convergence of approximate solutions for the minimal surface equation

$$
\sum_{i=1}^{n} \frac{\partial}{\partial x_{i}}\left(\frac{f_{x_{i}}}{\sqrt{1+|\nabla f|^{2}}}\right)=0
$$

in domain $\Omega$ subject to the boundary condition

$$
\left.f\right|_{\partial \Omega}=\left.\varphi\right|_{\partial \Omega},
$$

where $\varphi \in C(\bar{\Omega})$. It should be noted that this Dirichlet problem is not solvable for an arbitrary domain (even with a smooth boundary). For planar domains the necessary and sufficient condition for the solvability of the Dirichlet problem for an arbitrary continuous boundary function $\varphi(x)$ is the convexity of this domain. In the space of dimension greater than two, such condition is the non-negativity of the mean curvature of the boundary w.r.t. the outward normal. Precise formulation and the proof of these results can be found in works [3]-[10]. In the present work we impose no conditions for domain $\Omega$, but we assume that for a given boundary

A.A. Klyachin, I.V.Truhlyaeva, On COnVERgence of Polynomial SOlutions of minimal Surface.

(c) Klyachin A.A., Truhlyaeva I.V. 2016.

The work is supported by RFBR (project no. 15-41-02517-r_povolzhe_a.

Submitted May 15, 2015. 
function $\varphi(x)$ problem (1)-(2) is solvable. It is clear that for an arbitrary domain $\Omega$, such functions $\varphi(x)$ exist.

We study the issue on uniform convergence of polynomial approximate solutions to the minimal surface equation constructed by means of algebraic polynomials. In work [11] a similar convergence problem for piece-wise linear approximate solution to boundary value problem (1)(2) was solved, while in work 12 there was given a description of numerical realization of finite elements methods based on piece-wise linear functions. Let us provide required definitions.

Suppose that $\Omega \subset \mathbb{R}^{2}$ is a bounded domain such that some polynomial $\psi(x, y)$ of degree at most $N_{0}$ in each variable satisfies $\psi(x, y)=0$ as $(x, y) \in \partial \Omega$ and $\psi(x, y)>0$ as $(x, y) \in \Omega$. For a natural $N$ we denote by $L_{N}$ the set of all polynomials of the form

$$
v_{N}(x, y)=\psi(x, y) \sum_{n, m=1}^{N} c_{n m} x^{n} y^{m}
$$

It is clear that $v_{N}(x, y)=0$ for $(x, y) \in \partial \Omega$. Assume that $\varphi \in C^{1}(\Omega)$. Consider the problem on finding a polynomial $v_{N}^{*}$ on which the area integral

$$
\sigma\left(\varphi+v_{N}\right)=\iint_{\Omega} \sqrt{1+\left|\nabla \varphi+\nabla v_{N}\right|^{2}} d x d y \rightarrow \min , \quad v_{N} \in L_{N} .
$$

attains its minimum. It is easy to show that solution $v_{N}^{*}$ to problem (3) satisfies the identity

$$
\iint_{\Omega} \frac{\left\langle\nabla \varphi+\nabla v_{N}^{*}, \nabla v_{N}\right\rangle}{\sqrt{1+\left|\nabla \varphi+\nabla v_{N}^{*}\right|^{2}}} d x d y=0 \quad \forall v_{N} \in L_{N}
$$

Theorem 1. Problem (3) is uniquely solvable.

Proof. It is clear the area $\sigma\left(\varphi+v_{N}\right)$ is a function $\sigma\left(c_{11}, c_{12}, \ldots, c_{N N}\right)$ of finitely many variables $c_{n m}, n, m=1, \ldots, N$. This function is obviously continuous. At that,

$$
\lim _{|c| \rightarrow \infty} \sigma\left(c_{n m}\right)=+\infty,
$$

where $|c|=\max _{1 \leqslant n, m \leqslant N}\left|c_{n m}\right|$. Therefore, there exists a set of numbers $c_{n m}^{*}$, at which function $\sigma\left(c_{11}, c_{12}, \ldots, c_{N N}\right)$ attains its minimal value. We denote by $v_{N}^{*}$ the polynomial associated with this set of coefficients. It satisfies condition (4).

Let us show the uniqueness. Assume that there exists one more function $v_{N}^{1} \in L_{N}$ being a solution to problem (3). It also satisfies identity (4). Deducting one identity from the other for $v_{N}=v_{N}^{*}-v_{N}^{1}$, we obtain

$$
\iint_{\Omega}\left(\frac{\left\langle\nabla f^{*}, \nabla f^{*}-\nabla f^{1}\right\rangle}{\sqrt{1+\left|\nabla f^{*}\right|^{2}}}-\frac{\left\langle\nabla f^{1}, \nabla f^{*}-\nabla f^{1}\right\rangle}{\sqrt{1+\left|\nabla f^{1}\right|^{2}}}\right) d x d y=0
$$

where $f^{*}=\varphi+v_{N}^{*}, f^{1}=\varphi+v_{N}^{1}$.

We shall make use of the identity

$$
\left\langle\frac{\xi}{\sqrt{1+|\xi|^{2}}}-\frac{\eta}{\sqrt{1+|\eta|^{2}}}, \xi-\eta\right\rangle \geqslant \frac{|\xi-\eta|^{2}}{\sqrt{1+|\xi|^{2}}\left(\sqrt{1+|\xi|^{2}} \sqrt{1+|\eta|^{2}}+|\xi||\eta|+1\right)},
$$

which holds for all vectors $\xi, \eta \in \mathbb{R}^{2}$ and it can be obtained as follows. We first observe that

$$
\sqrt{1+|\xi|^{2}} \geqslant \sqrt{1+|\eta|^{2}}+\frac{\langle\eta, \xi-\eta\rangle}{\sqrt{1+|\eta|^{2}}}
$$


Then

$$
\begin{aligned}
\left\langle\frac{\xi}{\sqrt{1+|\xi|^{2}}}-\frac{\eta}{\sqrt{1+|\eta|^{2}}}, \xi-\eta\right\rangle & =-\frac{\langle\xi, \eta-\xi\rangle}{\sqrt{1+|\xi|^{2}}}-\frac{\langle\eta, \xi-\eta\rangle}{\sqrt{1+|\eta|^{2}}} \\
& \geqslant \sqrt{1+|\eta|^{2}}-\sqrt{1+|\xi|^{2}}-\frac{\langle\xi, \eta-\xi\rangle}{\sqrt{1+|\xi|^{2}}} \\
& =\frac{\sqrt{1+|\xi|^{2}} \sqrt{1+|\eta|^{2}}-\langle\xi, \eta\rangle-1}{\sqrt{1+|\xi|^{2}}} \\
& \geqslant \frac{|\xi-\eta|^{2}}{\sqrt{1+|\xi|^{2}}\left(\sqrt{1+|\xi|^{2}} \sqrt{1+|\eta|^{2}}+|\xi||\eta|+1\right)}
\end{aligned}
$$

Thus, it follows from inequalities (6) and identity (5) that

$$
\iint_{\Omega} \frac{\left|\nabla f^{*}-\nabla f^{1}\right|^{2}}{\sqrt{1+\left|\nabla f^{*}\right|^{2}}\left(\sqrt{1+\left|\nabla f^{*}\right|^{2}} \sqrt{1+\left|\nabla f^{1}\right|^{2}}+\left|\nabla f^{*}\right|\left|\nabla f^{1}\right|+1\right)} d x d y \leqslant 0 .
$$

Hence, $\nabla f^{*} \equiv \nabla f^{1}$. This yields that $\nabla v_{N}^{*} \equiv \nabla v_{N}^{1}$. Since polynomials $v_{N}^{*}$ and $v_{N}^{1}$ vanish on boundary $\partial \Omega$, we have $v_{N}^{*}(x, y)=v_{N}^{1}(x, y)$ for all $(x, y) \in \Omega$. The uniqueness is proven.

Definition. Function $f^{*}=\varphi+v_{N}^{*}, v_{N}^{*} \in L_{N}$ is called a polynomial solution to boundary value problem (1) -(2) if identity (4) holds true for each polynomial.

In what follows we shall be interested in the issue on uniform convergence of a sequence of polynomial solutions $\varphi+v_{N}^{*}$ as $N \rightarrow \infty$. First of all we shall show that under certain conditions, the gradients of these functions are bounded by a constant independent of $N$. This property will allow us to obtain an estimate for the rate of uniform convergence to the exact solution.

\section{Estimate For the GRAdiEnt of a POLYNOMial SOlUtion}

Let $f \in C^{2}(\Omega) \cap C(\bar{\Omega})$ be a solution to problem (1)-(2). We introduce quantity $\delta(\xi, \eta)$ for arbitrary vectors $\xi, \eta \in \mathbb{R}^{2}$

$$
\delta(\xi, \eta)=\sqrt{1+|\eta|^{2}}-\sqrt{1+|\xi|^{2}}-\frac{\langle\xi, \eta-\xi\rangle}{\sqrt{1+|\xi|^{2}}} .
$$

It is easy to see that $\delta(\xi, \eta)>0$ for all $\xi \neq \eta$. We shall also make use of the following polynomial characteristics of a domain:

$$
\lambda_{N}=\inf _{v \in L_{N}} \frac{\left(\iint_{\Omega}|\nabla v|^{2} d x d y\right)^{1 / 2}}{\sqrt{|\Omega|} \sup _{\Omega}|\nabla v|}>0,
$$

where $|\Omega|$ is the area of domain $\Omega$. It is clear that $0<\lambda_{N} \leqslant 1$. We shall estimate the rate of convergence of $\lambda_{N}$ to zero as $N \rightarrow \infty$ in the fifth section of the present paper.

Letting $\xi=\nabla f, \eta=\nabla \varphi+\nabla v_{N}^{*}$ and employing equation (1), we obtain

$$
\iint_{\Omega} \delta\left(\nabla f, \nabla \varphi+\nabla v_{N}^{*}\right) d x d y=\sigma\left(\varphi+v_{N}^{*}\right)-\sigma(f) .
$$

Employing the inequality (see (6))

$$
\sqrt{1+|\eta|^{2}}-\sqrt{1+|\xi|^{2}}-\frac{\langle\xi, \eta-\xi\rangle}{\sqrt{1+|\xi|^{2}}} \geqslant \frac{|\xi-\eta|^{2}}{\sqrt{1+|\xi|^{2}}\left(\sqrt{1+|\xi|^{2}} \sqrt{1+|\eta|^{2}}+|\xi||\eta|+1\right)}
$$


we conclude that

$$
\begin{aligned}
& \iint_{\Omega} \frac{\left|\nabla f-\nabla \varphi-\nabla v_{N}^{*}\right|^{2} d x d y}{\sqrt{1+|\nabla f|^{2}}\left(\sqrt{1+|\nabla f|^{2}} \sqrt{1+\left|\nabla \varphi+\nabla v_{N}^{*}\right|^{2}}+|\nabla f|\left|\nabla \varphi+\nabla v_{N}^{*}\right|+1\right)} \\
& \leqslant \sigma\left(\varphi+v_{N}^{*}\right)-\sigma(f) .
\end{aligned}
$$

By the previous inequality we obtain

$$
\iint_{\Omega} \frac{\left|\nabla f-\nabla \varphi-\nabla v_{N}^{*}\right|^{2} d x d y}{\sqrt{1+\left|\nabla \varphi+\nabla v_{N}^{*}\right|^{2}}} \leqslant 3\left(1+P_{0}^{2}\right)\left(\sigma\left(\varphi+v_{N}^{*}\right)-\sigma(f)\right) .
$$

We let $K=\max \left\{\sup _{\Omega}|\nabla \varphi|, 1\right\}$ and $A_{N}=\sup _{\Omega}\left|\nabla v_{N}^{*}\right|$. Then

$$
\iint_{\Omega}\left|\nabla f-\nabla \varphi-\nabla v_{N}^{*}\right|^{2} d x d y \leqslant 3 \sqrt{1+2 K^{2}+2 A_{N}^{2}}\left(1+P_{0}^{2}\right)\left(\sigma\left(\varphi+v_{N}^{*}\right)-\sigma(f)\right) .
$$

We introduce the notation $g=f-\varphi$. It is clear that $g(x, y)=0$ as $(x, y) \in \partial \Omega$. Given an arbitrary function $h \in C(\bar{\Omega})$, by $h_{N}$ we denote some approximation of function $h$ by the functions in space $L_{N}$. The way how to do this approximation is not important and it will be specified in the next section. Employing the above notation, we have

$$
\begin{aligned}
\left(\iint_{\Omega}\left|\nabla f-\nabla \varphi-\nabla v_{N}^{*}\right|^{2} d x d y\right)^{1 / 2} \geqslant & \left(\iint_{\Omega}\left|\nabla g_{N}-\nabla v_{N}^{*}\right|^{2} d x d y\right)^{1 / 2} \\
& -\left(\iint\left|\nabla g-\nabla g_{N}\right|^{2} d x d y\right)^{1 / 2}
\end{aligned}
$$

Hence, since $g_{N}-v_{N}^{*} \in L_{N}$, we get

$$
\begin{aligned}
\sup _{\Omega}\left|\nabla g_{N}-\nabla v_{N}^{*}\right| \leqslant & \frac{1}{\sqrt{|\Omega|} \lambda_{N}}\left(\iint_{\Omega}\left|\nabla g-\nabla g_{N}\right|^{2} d x d y\right)^{1 / 2} \\
& +\frac{1}{\sqrt{|\Omega|} \lambda_{N}}\left(\iint_{\Omega}\left|\nabla g-\nabla v_{N}^{*}\right|^{2} d x d y\right)^{1 / 2} \\
\leqslant & \frac{1}{\sqrt{|\Omega|} \lambda_{N}}\left(\iint_{\Omega}\left|\nabla g-\nabla g_{N}\right|^{2} d x d y\right)^{1 / 2}+ \\
& +\frac{\left(3\left(1+P_{0}^{2}\right) \sqrt{1+2 K^{2}+2 A_{N}^{2}}\right)^{1 / 2}}{\sqrt{|\Omega|} \lambda_{N}} \sqrt{\sigma\left(\varphi+v_{N}^{*}\right)-\sigma(f) .}
\end{aligned}
$$

We employ the following inequality

$$
\frac{x}{\sqrt[4]{a+x^{2}}} \geqslant \sqrt[4]{a+x^{2}}-\frac{\sqrt{a}}{\sqrt[4]{a+x^{2}}}
$$


which holds for $x \geqslant 0$ and $a>0$. Letting $x=\sqrt{2} A_{N}, a=1+2 K^{2}$, by this inequality we obtain

$$
\begin{aligned}
A_{N} \leqslant & \sup _{\Omega}\left|\nabla g_{N}\right|+\frac{1}{\sqrt{|\Omega|} \lambda_{N}}\left(\iint_{\Omega}\left|\nabla g-\nabla g_{N}\right|^{2} d x d y\right)^{1 / 2} \\
& +\left(\frac{3\left(1+P_{0}^{2}\right)}{|\Omega| \lambda_{N}^{2}}\left(\sigma\left(\varphi+v_{N}^{*}\right)-\sigma(f)\right)\right)^{1 / 2}+1
\end{aligned}
$$

Since function $v_{N}^{*}$ is a solution to problem (3), we arrive at the estimate

$$
\begin{aligned}
\sup _{\Omega}\left|\nabla v_{N}^{*}\right| \leqslant & \sup _{\Omega}\left|\nabla g_{N}\right|+\frac{1}{\sqrt{|\Omega|} \lambda_{N}}\left(\iint_{\Omega}\left|\nabla g-\nabla g_{N}\right|^{2} d x d y\right)^{1 / 2} \\
& +\left(\frac{3\left(1+P_{0}^{2}\right)}{|\Omega| \lambda_{N}^{2}}\left(\sigma\left(\varphi+g_{N}\right)-\sigma(f)\right)\right)^{1 / 2}+1 .
\end{aligned}
$$

In order to obtain the final inequality, we note that function $\varphi+g=f$ solves equation (1) and hence, $a^{\prime}(0)=0$, where $a(t)=\sigma\left(f+t\left(\varphi+g_{N}-f\right)\right)$. Letting $f^{t}=f+t\left(\varphi+g^{N}-f\right)$, we have

$$
\begin{aligned}
\sigma\left(\varphi+g_{N}\right) & -\sigma(f)=\int_{0}^{1} d s \int_{0}^{s} a^{\prime \prime}(t) d t \\
& =\int_{0}^{1} d s \int_{0}^{s} d t \iint_{\Omega} \frac{\left(1+\left|\nabla f^{t}\right|^{2}\right)\left|\nabla f-\nabla\left(\varphi+g_{N}\right)\right|^{2}-\left\langle\nabla f^{t}, \nabla f-\nabla\left(\varphi+g_{N}\right)\right\rangle^{2}}{\left(1+\left|\nabla f^{t}\right|^{2}\right)^{3 / 2}} d x d y \\
& \leqslant \iint_{\Omega}\left|\nabla f-\nabla\left(\varphi+g_{N}\right)\right|^{2} d x d y=\iint_{\Omega}\left|\nabla g-\nabla g_{N}\right|^{2} d x d y .
\end{aligned}
$$

Then by inequality (8) we arrive at the estimate

$$
\sup _{\Omega}\left|\nabla v_{N}^{*}\right| \leqslant 1+\sup _{\Omega}\left|\nabla g_{N}\right|+\frac{1+\sqrt{3\left(1+P_{0}^{2}\right)}}{\sqrt{|\Omega|} \lambda_{N}}\left(\iint_{\Omega}\left|\nabla g-\nabla g_{N}\right|^{2} d x d y\right)^{1 / 2} .
$$

Thus, we have proved the following theorem.

Theorem 2. Let $f$ be a solution to equation (1) satisfying boundary condition (2). If $v_{N}^{*}(x, y) \in L_{N}$ is a solution to problem (3), its gradient satisfies estimate (9).

Remark. It follows from Theorem 2 that as $N \rightarrow \infty$, the gradients of approximated solutions $\varphi+v_{N}^{*}$ are uniformly bounded if the same is true for

$$
\frac{1}{\lambda_{N}}\left(\iint_{\Omega}\left|\nabla g-\nabla g_{N}\right|^{2} d x d y\right)^{1 / 2}
$$

In this case the gradients of functions $g_{N}(x, y)$ are also bounded. To clarify these issues we need to estimate the rate of approximation of function $g$ by polynomials $g_{N}$ and to find out the behavior of sequence $\lambda_{N}$ as $N \rightarrow \infty$.

\section{Approximation of SMOOTh FUnCTIONS BY POLYNOMIALS}

Let $\Omega$ be a bounded domain with a boundary $\Gamma, k$ be a natural number and function $\psi(x, y)$ satisfy the conditions: 
1) function $\psi$ is $k$ times differentiable and its derivatives of $k$ th order satisfy the Lipschitz condition;

2) $\psi(x, y)=0$ as $(x, y) \in \Gamma$ and $\psi(x, y) \neq 0$ as $(x, y) \in \mathbb{R}^{2} \backslash \Gamma$;

3) $|\nabla \psi(x, y)|>0$ as $(x, y) \in \Gamma$.

Then, as it was shown in paper [13], given a function $u(x, y)$, which is $k$ times continuously differentiable in $\bar{\Omega}$ and vanishes at $\Gamma$, we can find a sequence of polynomials $P_{N}(x, y)$ of degree $\leqslant N$ in each variable $x, y$ such that

$$
\left\|u-\psi P_{N}\right\|_{C^{r}(\Omega)} \leqslant \frac{\delta_{N}(u)}{N^{k-r}}, r=0,1, \ldots, k,
$$

where $\delta_{N}(u) \rightarrow 0$ as $N \rightarrow \infty$.

Hereafter we assume that $g=f-\varphi \in C^{k}(\Omega)$. We specify the way of choosing function $g_{N}$ : we let $g_{N}=\psi P_{N}$, where approximating polynomial is chosen for function $g=f-\varphi$. Applying for $r=1$ estimates (10) for $u=f-\varphi$ in inequality (9), we obtain

$$
\sup _{\Omega}\left|\nabla v_{N}^{*}\right| \leqslant 1+K+P_{0}+\frac{2+\sqrt{3\left(1+P_{0}^{2}\right)}}{\lambda_{N}} \frac{\delta_{N}(f-\varphi)}{N^{k-1}} .
$$

By this inequality we see that the gradients of approximate solutions are bounded by a constant independent of $N$ as $N \rightarrow \infty$ if $\lambda_{N}$ tends to zero not faster than $O\left(1 / N^{k-1}\right)$. In the next section we shall study this issue.

\section{ESTIMATE FOR $\lambda_{N}$}

Let us provide an example of a lower bound for $\lambda_{N}$. It is clear that

$$
\lambda_{N}=\inf _{v \in L_{N}} \frac{\left(\iint_{\Omega}|\nabla v|^{2} d x d y\right)^{1 / 2}}{\sqrt{|\Omega|} \sup _{\Omega}|\nabla v|} \geqslant \inf _{P} \frac{\left(\iint_{\Omega}|\nabla P|^{2} d x d y\right)^{1 / 2}}{\sqrt{|\Omega|} \sup _{\Omega}|\nabla P|},
$$

where the infimum is taken over all polynomials of degree at most $N^{\prime}=N+N_{0}$ in each variable.

We shall make use of the following inequality by A.A. Markov (see, for instance [14, Sect. $6]$ ) for a polynomial $P(x)$ of one variable of degree $N$ on a segment $[a, b]$

$$
\left|P^{\prime}(x)\right| \leqslant \frac{2 N^{2}}{b-a} \max _{[a, b]}|P(x)| .
$$

It implies easily a similar inequality for a rectangle in the case of polynomial of two variables. Let $P(x, y)$ be a polynomial, whose degree in each variable is at most $N$. We let

$$
M=\max _{[a, b] \times[c, d]}|P(x, y)| .
$$

Then by A.A. Markov inequality in each variable we have

$$
\left|\frac{\partial P}{\partial x}\right| \leqslant \frac{2 N^{2}}{b-a} \max _{[a, b]}|P(x, y)| \leqslant M \frac{2 N^{2}}{b-a}, \quad\left|\frac{\partial P}{\partial y}\right| \leqslant M \frac{2 N^{2}}{d-c} .
$$

Therefore,

or

$$
|\nabla P|^{2} \leqslant 4 N^{4} M^{2} \frac{(d-c)^{2}+(b-a)^{2}}{(b-a)^{2}(d-c)^{2}}
$$

$$
|\nabla P| \leqslant 2 N^{2} M \frac{\sqrt{(d-c)^{2}+(b-a)^{2}}}{(b-a)(d-c)}=2 N^{2} M \frac{d}{S},
$$

where $d$ is the diagonal of the rectangle, and $S$ is its area. We consider this inequality for a square with side $a>0$. Then $d=a \sqrt{2}, S=a^{2}$. This is why

$$
\left|\nabla P_{N}\right| \leqslant N^{2} M \frac{2 \sqrt{2}}{a} \text {. }
$$


It is clear that this inequality is true for each square not necessarily having sides parallel to the axes. We let

$$
\inf _{P} \frac{\left(\iint_{\Omega}|P|^{2} d x d y\right)^{1 / 2}}{\sqrt{\Omega} \sup _{\Omega}|P|}=\widetilde{\lambda}_{N}
$$

where the infimum is taken over all polynomials of degree at most $N$ in each variable. We first find the estimate of $\widetilde{\lambda}_{N}$ for a square $K$ with side $a>0$. Let $z=(x, y) \in K$ and $z_{0} \in K$ is such that $P\left(z_{0}\right)=\max _{K}|P|=M$. Then

$$
P\left(z_{0}\right)-P(z) \leqslant\left|z-z_{0}\right| \max _{K}|\nabla P| \leqslant N^{2} \frac{2 \sqrt{2}}{a} M\left|z-z_{0}\right| .
$$

If $\left|z-z_{0}\right|<a /\left(4 \sqrt{2} N^{2}\right)$, then $M-P(z) \leqslant \frac{M}{2}$. Thus, $P(x, y) \geqslant \frac{M}{2}$ for $(x, y) \in K \cap B_{\frac{a}{4 \sqrt{2} N^{2}}}\left(z_{0}\right)$. Then

$$
\iint_{K} P^{2}(x, y) d x d y \geqslant \iint_{K \cap B} P_{\frac{a}{4 \sqrt{2} N^{2}}\left(z_{0}\right)} P^{2}(z) d x d y \geqslant \frac{M^{2}}{4} \iint_{K \cap B} d x d y \geqslant \frac{M^{2}}{16} \pi \frac{a^{2}}{32 N^{4}} .
$$

This is why

$$
\left(\iint_{K} P^{2}(x, y) d x d y\right)^{\frac{1}{2}} \geqslant \frac{M a}{16 \sqrt{2} N^{2}} \sqrt{\pi}
$$

Since $P$ is an arbitrary admissible polynomial, then

$$
\widetilde{\lambda}_{N} \geqslant \frac{1}{16} \sqrt{\frac{\pi}{2}} \frac{1}{N^{2}}
$$

We apply the proven inequality for partial derivative of polynomial $P$, which are also polynomials. Therefore,

$$
\left(\iint_{K} P_{x}^{2}(x, y) d x d y\right)^{\frac{1}{2}} \geqslant \widetilde{\lambda}_{N} \max _{K}\left|P_{x}\right| \sqrt{|K|}, \quad\left(\iint_{K} P_{y}^{2}(x, y) d x d y\right)^{\frac{1}{2}} \geqslant \widetilde{\lambda}_{N} \max _{K}\left|P_{y}\right| \sqrt{|K|} .
$$

It yields

$$
|\nabla P|^{2} \geqslant \widetilde{\lambda}_{N}^{2}|K|\left(\left(\max _{K}\left|P_{x}\right|\right)^{2}+\left(\max _{K}\left|P_{y}\right|\right)^{2}\right) \geqslant \widetilde{\lambda}_{N}^{2}|K|\left(\max _{K}\left|P_{x}\right|^{2}+\left|P_{y}\right|^{2}\right)=|K|\left(\left(\max _{K}|\nabla P|\right)^{2} .\right.
$$

Hence,

Therefore,

$$
\frac{\left(\iint_{K}|\nabla P|^{2} d x d y\right)^{\frac{1}{2}}}{|K| \max _{K}|\nabla P|} \geqslant \widetilde{\lambda}_{N} \geqslant \frac{1}{16 N^{2}} \sqrt{\frac{\pi}{2}}
$$

$$
\lambda_{N} \geqslant \frac{1}{16 N^{2}} \sqrt{\frac{\pi}{2}}
$$

Thus, we have obtained the lower bound for $\lambda_{N}$ in the case when $\Omega$ is a square with side $a>0$. We observe that this estimate is independent of the size of square. Employing inequality (12), we obtain the estimate for an arbitrary planar domain $\Omega$.

For each $z_{0} \in \Omega$ there exists a maximal square $K \subset \bar{\Omega}$ with sides not necessarily parallel to the axes such that $z_{0} \in K$. Let the side of the square be $a\left(z_{0}\right)>0$. We denote

$$
\Delta(\Omega)=\inf _{z_{0} \in \Omega} a\left(z_{0}\right) .
$$


We shall assume that $\Delta(\Omega)>0$. For instance, if $\Omega$ is a ball of radius $R>0$, it is easy to see that for each point $z_{0}$ in this ball we have $a\left(z_{0}\right)=R \sqrt{2}$. This is why for the ball we have $\Delta(\Omega)=R \sqrt{2}$. As another example, we provide a domain inside the ellipse

$$
\Omega=\left\{(x, y): \frac{x^{2}}{a^{2}}+\frac{y^{2}}{b^{2}}<1\right\}, a>b .
$$

At the point with the coordinates $(a, 0)$, the ellipse has the minimal curvature radius, which is equal to $b^{2} / a$. It is easy to obtain that each point $z_{0} \in \Omega$ lies inside some ball of radius $b^{2} / a$ lying inside the ellipse. This is why for the ellipse we have $\Delta(\Omega) \geqslant \sqrt{2} b^{2} / a$.

It is easy to estimate $\lambda_{N}$ via $\Delta(\Omega)$. Indeed, let $z_{0} \in \Omega$ be such that $\max _{\Omega}|\nabla P|=\left|\nabla P\left(z_{0}\right)\right|$ and square $K \subset \bar{\Omega}$ contain this point. Then

$$
\sqrt{\iint_{\Omega}|\nabla P(x, y)|^{2} d x d y} \geqslant \sqrt{\iint_{K}|\nabla P(x, y)|^{2} d x d y} \geqslant \sqrt{\frac{\pi}{2}} \frac{1}{16 N^{2}} \sqrt{|K|} \max _{K}|\nabla P| .
$$

It implies easily the inequality

$$
\lambda_{N} \geqslant \frac{\Delta(\Omega)}{16 N^{2} \sqrt{|\Omega|}} \sqrt{\frac{\pi}{2}} .
$$

Hence, if domain $\Omega$ is such that each its point can be put inside a square $K \subset \bar{\Omega}$ with side $\Delta(\Omega)>0$, then estimate 13 holds true. It is clear that if the domain has a smooth boundary, then $\Delta(\Omega)>0$. For more general domains we first obtain the estimate of $\lambda_{N}$ for a rhombus.

Assume that in plane with Cartesian coordinates $(x, y)$ we are given a rhombus $R$ with vertices at the points

$$
(0,0), \quad(a, 0), \quad(a \cos \alpha, a \sin \alpha), \quad(a(1+\cos \alpha), a \sin \alpha),
$$

where $\alpha \in(0, \pi / 2], a>0$. By means of the linear transform

$$
u=x \sin \alpha-y \cos \alpha, \quad v=y
$$

on the plane with coordinates $(u, v)$ we obtain square $K$ with the vertices

$$
(0,0), \quad(a \sin \alpha, 0), \quad(0, a \sin \alpha), \quad(a \sin \alpha, a \sin \alpha),
$$

Now let $P=P(x, y)$ be an arbitrary polynomial, whose degree in each variable does not exceed $N$. It is easy to see that

$$
P_{x}^{2}+P_{y}^{2}=\left(P_{u} \sin \alpha\right)^{2}+\left(-P_{u} \cos \alpha+P_{v}\right)^{2} \geqslant(1-\cos \alpha)\left(P_{u}^{2}+P_{v}^{2}\right) .
$$

Then it follows from (12) that

$$
\begin{aligned}
\frac{\left(\iint_{R}\left(P_{x}^{2}+P_{y}^{2}\right) d x d y\right)^{1 / 2}}{\sqrt{|R|} \max _{R}|\nabla P|} \geqslant \frac{\sqrt{1-\cos \alpha}}{\sin \alpha} \cdot \frac{\left(\iint_{K}\left(P_{u}^{2}+P_{v}^{2}\right) d u d v\right)^{1 / 2}}{\sqrt{|R|} \max _{K}|\nabla P|} \\
=\frac{\sqrt{1-\cos \alpha}}{\sin \alpha} \cdot \frac{\left(\iint_{R}\left(P_{u}^{2}+P_{v}^{2}\right) d u d v\right)^{1 / 2}}{\sqrt{|K|} \max _{K}|\nabla P|} \sqrt{\frac{|K|}{|R|}} \\
=\sqrt{\frac{1-\cos \alpha}{\sin \alpha}} \cdot \frac{\left(\iint_{R}\left(P_{u}^{2}+P_{v}^{2}\right) d u d v\right)^{1 / 2}}{\sqrt{|K|} \max _{K}|\nabla P|} \geqslant \sqrt{\frac{\pi(1-\cos \alpha)}{2 \sin \alpha}} \frac{1}{16 N^{2}} .
\end{aligned}
$$


Hence, for the rhombus with a side $a>0$ and an acute angle $\alpha \in(0, \pi / 2]$ the inequality

$$
\lambda_{N} \geqslant \sqrt{\frac{\pi(1-\cos \alpha)}{2 \sin \alpha}} \frac{1}{16 N^{2}}
$$

holds true.

Suppose that we are given a planar domain $\Omega$. We denote by $\alpha(\Omega) \in(0, \pi / 2]$ an angle such that each point of the domain is contained in the rhombus $R \subset \Omega$ with an acute angle $\alpha(\Omega)$. For each $z_{0} \in \Omega$ we find a rhombus $R \subset \bar{\Omega}$ with maximal side such that $z_{0} \in R$. Let the side of this rhombus is $a\left(z_{0}\right)>0$. We let

$$
\Delta_{1}(\Omega)=\inf _{z_{0} \in \Omega} a\left(z_{0}\right)>0 .
$$

Arguing as above, we obtain the inequality

$$
\lambda_{N} \geqslant \sqrt{\frac{\pi}{|\Omega|}} \cdot \frac{\Delta_{1}(\Omega) \sin (\alpha(\Omega) / 2)}{16 N^{2}} .
$$

Taking into consideration that the polynomials in $L_{N}$ are of degree at most $N_{0}+N$, we arrive at the following statement.

Theorem 3. Let a bounded domain $\Omega \subset \mathbb{R}^{2}$ be such that $\Delta_{1}(\Omega)>0$ and $\alpha(\Omega)>0$. Then the following estimate

$$
\lambda_{N} \geqslant \sqrt{\frac{\pi}{|\Omega|}} \cdot \frac{\Delta_{1}(\Omega) \sin (\alpha(\Omega) / 2)}{16\left(N+N_{0}\right)^{2}}
$$

holds true.

\section{Estimate FOR THE RATE OF UNIFORM CONVERGENCE}

We borrow the method of estimating the solutions from work [15]. Let $f$ be a solution to the minimal equation surface in domain $\Omega \subset \mathbb{R}^{2}, f \in C^{k}(\bar{\Omega})$. Let $v_{N}^{*}$ be a solution to problem (3) satisfying (4). We let $f_{N}^{*}=\varphi+v_{N}^{*}$.

We shall assume that

$$
\sup _{\Omega}|\nabla f|=P_{0}<+\infty
$$

We shall argue as in work [15]. We let $f^{t}(x, y)=f_{N}^{*}(x, y)+t\left(f(x, y)-f_{N}^{*}(x, y)\right)$ and $P_{N}^{*}=\sup _{\Omega}\left|\nabla f_{N}^{*}\right|, P_{N}=\max \left\{1, P_{0}, P_{N}^{*}\right\}$. It is clear that $\left.f^{*}\right|_{\partial \Omega}=\left.f\right|_{\partial \Omega}$. We observe that, generally speaking, $P_{N}$ depend on $N$. However, if we assume that $k>2$ and constants $\Delta_{1}(\Omega)$ and $\alpha(\Omega)$ for the domain, then it follows from inequalities (15) and (9) that as $N \rightarrow \infty$, quantity $P_{N}$ is bounded by some constant $P$.

Since as $t=0$ the function $\sigma(t)=\sigma\left(f^{t}\right)$ takes the minimal value, then $\sigma^{\prime}(0)=0$. Employing this identity, we obtain

$$
\begin{aligned}
\sigma\left(f_{N}^{*}\right)-\sigma(f) & =\int_{0}^{1} d s \int_{0}^{s} \sigma^{\prime \prime}(t) d t \\
& =\int_{0}^{1} d s \int_{0}^{s} d t \iint_{\Omega} \frac{\left(1+\left|\nabla f^{t}\right|^{2}\right)\left|\nabla f_{N}^{*}-\nabla f\right|^{2}-\left\langle\nabla f^{t}, \nabla f_{N}^{*}-\nabla f\right\rangle^{2}}{\left(1+\left|\nabla f^{t}\right|^{2}\right)^{3 / 2}} d x d y \\
& \geqslant \int_{0}^{1} d s \int_{0}^{s} d t \iint_{\Omega} \frac{\left|\nabla f-\nabla f_{N}^{*}\right|^{2}}{\left(1+\left|\nabla f^{t}\right|^{2}\right)^{3 / 2}} d x d y \geqslant \frac{1}{\sqrt{\left(1+P^{2}\right)^{3}}} \iint_{\Omega}\left|\nabla f-\nabla f_{N}^{*}\right|^{2} d x d y .
\end{aligned}
$$


We employ Poincaré inequality (see, for instance, [16, Subsect. 7.8]) for the function $h(x, y)=$ $f(x, y)-f_{N}^{*}(x, y),\left.h\right|_{\partial \Omega}=0$. By (17) we obtain

$$
\sigma\left(f_{N}^{*}\right)-\sigma(f) \geqslant \frac{\lambda(\Omega)}{\sqrt{\left(1+P^{2}\right)^{3}}} \iint_{\Omega}|h(x, y)|^{2} d x d y,
$$

where constant $\lambda(\Omega)=\pi /|\Omega|$ and $|\Omega|$ is the area of domain $\Omega$. We let $M=\sup _{\Omega}|h|$ and without loss of generality we can assume that there exists a point $z_{0}=\left(x_{0}, y_{0}\right) \in \Omega$, in which $h\left(x_{0}, y_{0}\right)=$ $M$. Let us show that $B_{M / 2 P}\left(z_{0}\right) \subset \Omega$. Indeed, let $z^{\prime} \in \partial \Omega$ be such that $\left|z_{0}-z^{\prime}\right|=\operatorname{dist}\left(z_{0}, \partial \Omega\right)$. Then

$$
2 P\left|z_{0}-z^{\prime}\right| \geqslant h\left(z_{0}\right)-h\left(z^{\prime}\right)=M-h\left(z^{\prime}\right)=M .
$$

Thus, the distance from point $z_{0}$ to boundary $\partial \Omega$ is greater than $M / 2 P$. Therefore, $B_{M / 4 P}\left(z_{0}\right) \subset$ $\Omega$. Suppose now that $z=(x, y) \in B_{M / 4 P}\left(z_{0}\right)$. Then

$$
h(z) \geqslant h\left(z_{0}\right)-2 P\left|z-z_{0}\right|>M-2 P \frac{M}{4 P}=M / 2 .
$$

We obtain that $B_{M / 4 P}\left(z_{0}\right) \subset D_{M}$, where

$$
D_{M}=\{(x, y) \in \Omega:|h(x, y)|>M / 2\} \subset \subset \Omega .
$$

This is why

$$
\begin{aligned}
\iint_{\Omega}|h(x, y)|^{2} d x d y & \geqslant \iint_{D_{M}}|h(x, y)|^{2} d x d y \\
& \geqslant \iiint_{B_{M / 4 P}\left(z_{0}\right)}\left(\frac{M}{2}\right)^{2} d x d y=\pi \frac{M^{2}}{4}\left(\frac{M}{4 P}\right)^{2}=\pi \frac{M^{4}}{64 P^{2}} .
\end{aligned}
$$

Thus,

$$
\max _{\Omega}\left|f-f_{N}^{*}\right| \leqslant \frac{4}{\sqrt{\pi}}\left(P^{5}|\Omega|\left(\sigma\left(f_{N}^{*}\right)-\sigma(f)\right)\right)^{1 / 4}
$$

Then we observe that function $v_{N}^{*}$ solves problem $(3)$. Hence, $\sigma\left(f_{N}^{*}\right)-\sigma(f) \leqslant \sigma\left(f-\varphi-g_{N}\right)-$ $\sigma(f)$. Employing the above proven inequality

$$
\sigma\left(f-\varphi-g_{N}\right)-\sigma(f) \leqslant \iint_{\Omega}\left|\nabla g-\nabla g_{N}\right|^{2} d x d y
$$

and estimate $(10)$ for $u=f-\varphi$, we arrive at the inequality

$$
\max _{\Omega}\left|f-f_{N}^{*}\right| \leqslant \frac{4}{\sqrt{\pi}}\left(P^{5}|\Omega| \frac{\delta_{N}^{2}(f-\varphi)}{N^{2 k-2}}\right)^{1 / 4} .
$$

We have proven the main result of the work.

Theorem 4. Assume that $f \in C^{k}(\bar{\Omega}), k \geqslant 3$, be a solution to the minimal surface equation (1) in domain $\Omega$, for which $\Delta_{1}(\Omega)>0$ and $\alpha(\Omega)>0$. Assume that this solution satisfies boundary condition (2) with a function $\varphi \in C^{k}(\bar{\Omega})$. Consider functions $v_{N}^{*} \in L_{N}$ being solutions to problem (3). Suppose that $P_{0}=\sup |\nabla f|<+\infty$ and $K=\sup |\nabla \varphi|<\infty$. Then the sequence of approximate solutions $f_{N}^{*}=\varphi+v_{N}^{*}$ converge uniformly to $f$ and the estimate

$$
\max _{\bar{\Omega}}\left|f-f_{N}^{*}\right| \leqslant \frac{4}{\sqrt{\pi}}\left(P^{5}|\Omega| \frac{\delta_{N}^{2}(f-\varphi)}{N^{2 k-2}}\right)^{1 / 4}
$$


holds true, where

$$
P=1+2 K+P_{0}+\frac{16 \sqrt{|\Omega|}\left(2+\sqrt{3\left(1+P_{0}^{2}\right)}\right)}{\sqrt{\pi} \Delta_{1}(\Omega) \sin (\alpha(\Omega) / 2)} \frac{\delta_{N}(f-\varphi)}{N^{k-1}}\left(N+N_{0}\right)^{2} .
$$

\section{BIBLIOGRAPHY}

1. S.G. Mikhlin. Variational methods in mathematical physics. Nauka, Moscow (1970). (in Russian).

2. L.V. Kantorovich, V.I. Krylov. Approximate methods of higher analysis. Nauka, Moscow (1962). (in Russian).

3. R. Finn. Remarks relevant to minimal surfaces and to surfaces of constant mean curvature // J. d'Analyse Math. 14:1, 139-160 (1965).

4. T. Rado. The problem of the least area and the problem of Plateau // Mathem. Zeit. 32:1, 763-796 (1930).

5. S.N. Bernshtein. On equations of calculus of variations // Uspekhi Matem. Nauk. 8, 32-74 (1941). (in Russian).

6. S.N. Bernshtein, I.G. Petrovskii. On the first boundary value problem (Dirichlet's problem) for equations of the elliptic type and on the properties of functions satisfying such equations // Uspekhi Matem. Nauk. 8, 8-31 (1941). (in Russian).

7. J. Serrin. The problem of Dirichlet for quasilinear elliptic differential equations with many independent variables // Phil. Trans. Royal Soc. London. A. 264:1153, 413-496 (1964).

8. G. Stampacchia. On some multiple integral problems in the calculus of variations // Comm. Pure Appl. Math. 16:4, 382-422 (1963).

9. H. Jenkins, J. Serrin. The Dirichlet problem for the minimal surface equation in higher dimension // J. Reine Angew. Math. 229, 170-187 (1968).

10. R.C. Bassanezi, U. Massari. The Dirichlet problem for the minimal surface equation in non-regular domains // Ann. Univ. Ferrara. 24:1, 181-189 (1978).

11. M.A. Gatsunaev, A.A. Klyachin. On uniform convergence of piecewise-linear solutions to minimal surface equation // Ufimskij Matem. Zhurn. 6:3, 3-16 (2014). [Ufa Math. J. 6:3, 3-16 (2014).]

12. A.A. Klyachin, V.A. Klyachin, E.G. Grigor'eva. Visualisation of calculating the form of minimal area surface // Scientific visualization. 6:2, 34-42 (2014). (in Russian).

13. I.Yu. Kharrik. On approximation of functions vanishing on the boundary of a region by functions of a special form // Matem. Sborn. 37(79):2, 353-384 (1955). (in Russian).

14. I.P. Natanson. Constructive theory of functions. Gostekhizdat, Moscow (1949). (in Russian).

15. A.A. Klyachin. On convergence rate of sequence providing minimum in variational problem // Bull. Volgograd State Univ. Ser. 1. Math. Phys. 16:1, 12-20 (2012). (in Russian).

16. D. Gilbarg, N.S. Trudinger. Elliptic partial differential equations of second order. Springer, Berlin (1983).

Alexei Alexandrovcih Klyachin,

Volgograd State University,

Universitetsky av., 100,

400062, Volgograd, Russia

E-mail: klyachin-aa@yandex.ru

Trukhlyaeva Irina Vladimirovna,

Volgograd State University,

Universitetsky av., 100,

400062, Volgograd, Russia

E-mail: irishka2027@mail.ru 